GI-MS48-P03

\section{Exploring structural biology on the web: to biologists and beyond}

David Armstrong ${ }^{1}$, Sameer Velankar ${ }^{1}$

1. PDBe, EMBL-EBI, Cambridge, United Kingdom

email: davida@ebi.ac.uk

Teaching macromolecular structure is often complicated by the need to have specialist tools to view structures and related information. At the PDBe we have integrated a number of different components that allow easy visualisation of structural data in different ways within the web browser.

These components include lightning-fast molecular visualisation with LiteMol and a sequence component to link this visualisation to the primary structure, via secondary structure topology diagrams. These interactive components enable students to explore sequence information and its relationship to $3 \mathrm{D}$ structure. The components integrate extra annotations including domains and validation data. Visualisation of electron density maps within LiteMol makes it an excellent tool for introducing students to the importance of validation in structural biology.

All of these components operate within a browser window, including on mobile devices, meaning that they are available to anyone, anywhere. This has enabled us to effectively teach macromolecular structure to students at all levels and even to introduce structural biology to artists and the wider general public. We hope this will help to bring structure to biology and beyond.

References:

Mir, S. et. al. (2018) Nucleic acids research, 46, D486-D492

Sehnal, D. et. al. (2017) Nature methods, 14, 1121-1122

Keywords: Visualisation, Sequence to structure, validation

\section{GI-MS48-P04}

\section{A model description of crystalline state under external influences}

Boris Kodess ${ }^{1}$, Pouline Kodess ${ }^{2}$

1. CMD, Materials Science - VNIIMS-ICS\&E, Moscow, Russia

2. Materials Science Dept. - ICS\&E, Aurora, CO, United States of America

email: kodess@mail.ru

A method is proposed for describing the change in the ground state of a phase of a crystalline substance under external influences. This manner of expression takes into account the multifactorial nature of governing factors which determine phase transitions and influence the functional characteristics of the materials being created from the substances. The phase space for crystals is constructed vector interactions) using three basic and enough independent factors and then associated with the composition of substance. They describe, first, the change in the level of available potential or the incoming energy to the substance; second, the time characteristics, included for describing emerging and existing phases; third, the spatial localization of the phase, respectively.

For a more detailed description of the processes of phase state change under external influences these factors consistently combine into the factors of the second level, which create subsystems to describe the origin of space-time patterns, the evolution of the state of the phase, and third, to describe the phase transitions in substances. Then a subsequent combination of all three specialized second-level factors in the third-level factors makes it possible to describe the relationship of changes in composition and structure with functional characteristics that reflect the generalized information nature of these characteristics; this can be used to describe more complex behavior, for example, temporal oscillations of all structural parameters in certain inorganic, usually multi-component substances.

The use of the model in educational practice has shown that the proposed method of description of a crystal phase state is useful for a more accessible and comprehensive, and also for a wider explanation of the multifactor nature of characteristics of condensed substances in the crystalline state. The model also ensures a better transfer of the accumulated knowledge about the specific features of the crystalline state into the processes of creating and developing new types of crystals and the technologies for the production and processing of materials, necessary for use in various spheres of life and industry.

The connection of this model with fundamental physico-chemical concepts can be useful for students as a new tool for deeper and successful mastering of crystallography. We noticed [1-3] that students whose crystallographic interests are related to the functional information properties of substances based on the key factors of the crystalline state, faster socialize in the modern information society and more confidently and reliably solve specific problems in the field of materials science. 